\title{
Nrf2 attenuates the innate immune response after experimental myocardial infarction
}

Daniel I. Bromage ${ }^{1}$, Silvia Trevelin ${ }^{1}$, Josef Huntington ${ }^{1}$, Victoria X. Yang ${ }^{1}$, Ananya

Muthukumar ${ }^{1}$, Sarah J. Mackie ${ }^{2}$, Greta Sawyer ${ }^{1}$, Xiaohong Zhang ${ }^{1}$, Celio X.C. Santos ${ }^{1}$, Niloufar

Safinia $^{3}$, loannis Smyrnias ${ }^{1,4}$, Mauro Giacca $^{1}$, Alex Ivetic ${ }^{1}$, Ajay M. Shah ${ }^{1}$

${ }^{1}$ School of Cardiovascular Medicine and Sciences, King's College London British Heart Foundation

Centre of Excellence, James Black Centre, 125 Coldharbour Lane, London, SE5 9NU, UK.

${ }^{2}$ School of Cancer and Pharmaceutical Sciences, SGDP Centre, King's College London, Memory Lane, London, SE5 8AF, UK.

${ }^{3}$ MRC Centre for Transplantation, Division of Transplantation Immunology and Mucosal Biology,

King's College London, James Black Centre, 125 Coldharbour Lane, London, SE5 9NU, UK.

${ }^{4}$ School of Veterinary Medicine, Faculty of Health and Medical Sciences, University of Surrey, Surrey,

GU2 7AL, UK.

Corresponding author: Dr Daniel Bromage, School of Cardiovascular Medicine and Sciences, King's College London British Heart Foundation Centre of Excellence, James Black Centre, 125 Coldharbour Lane, London, SE5 9NU, UK; Tel: +44 (0)20 7848 5189; e-mail: daniel.bromage@kcl.ac.uk; Twitter: @BromageDan.

Total word count (including abstract, figure legend and references): 4099

Tweet: Nrf2 signalling in leukocytes may be a target to prevent post-MI ventricular remodeling \#cardioimmunology \#heart failure.

Relationship with industry: MG is a member of the Scientific Advisory Boards of Trizell Holding SA, Lausanne and DINAQR AG, Zurich-London and is founder, consultant and equity holder in Purespring Therapeutics and Forcefield Therapeutics, London, which operate in the field of gene therapy and cardioprotection. AMS reports an advisory board association with Forcefield Therapeutics, London.

Sources of funding: DB has received funding from an NIHR Clinical Lectureship (CL-2016-17-001) and the Academy of Medical Sciences (SGL020 1087). AMS is supported by the British Heart Foundation 
bioRxiv preprint doi: https://doi.org/10.1101/2022.01.10.475615; this version posted January 10, 2022. The copyright holder for this preprint (which was not certified by peer review) is the author/funder. All rights reserved. No reuse allowed without permission.

(CH/1999001/11735, RE/18/2/34213, RM/17/3/33381). $M G$ is supported by the British Heart Foundation (RG/19/11/34633) and the European Research Council (Advanced Grant 787971 "CuRE”). 


\section{Abstract}

\section{Objectives}

We aimed to investigate the contribution of the transcription factor nuclear factor erythroidderived 2-like 2 (Nrf2) to the inflammatory response after experimental myocardial infarction (MI).

\section{Background}

There is compelling evidence implicating dysregulated inflammation in the mechanism of ventricular remodeling and heart failure (HF) after MI. The transcription factor Nrf2 (encoded by $\mathrm{Nfe} 2 / 2$ ) is a promising target in this context. It impedes transcriptional upregulation of pro-inflammatory cytokines and is anti-inflammatory in various murine models.

\section{Methods}

We subjected $\mathrm{Nrf2}^{-/-}$mice and wild type (WT) controls to permanent left coronary artery (LCA) ligation. The inflammatory response was investigated with fluorescence-activated cell sorting (FACS) analysis of peripheral blood and heart cell suspensions, together with qRTPCR of infarcted tissue for chemokines and their receptors. To investigate whether Nrf2mediated transcription is a dedicated function of leukocytes, we interrogated publicly available RNA-sequencing (RNA-seq) data from mouse hearts after permanent LCA ligation for Nrf2-regulated gene (NRG) expression.

Results

FACS analysis demonstrated a profoundly inflamed phenotype in the hearts of global $\mathrm{Nrf2}^{-1}$ mice as compared to WT mice after MI. Moreover, infarcted tissue from $\mathrm{Nrf2}^{-1}$ mice displayed higher expression of inflammatory cytokines, chemokines, and their receptors, including IL6, CCI2, and CxCr4. RNA-seq analysis showed upregulated NRG expression in WT mice after $\mathrm{Ml}$ compared to untreated mice, which was significantly higher in bioinformatically isolated $\mathrm{CCR} 2^{+}$cells. 


\section{Conclusions}

Taken together, the results suggest that Nrf2 signalling in leukocytes, and possibly CCR2 ${ }^{+}$ monocyte-derived cardiac resident macrophages, may be potential targets to prevent postMI ventricular remodeling.

\section{Keywords}

Nrf2, macrophage, neutrophil, myocardial infarction, remodeling. 


\section{Introduction}

Despite timely reperfusion, myocardial infarction (MI) is the commonest cause of heart failure (HF), and mortality remains high in such patients (1-5). HF after $\mathrm{Ml}$ is caused by ventricular remodeling, characterised by fibrosis, ventricular dilatation, and progressive functional deterioration. Post-MI inflammation is vital to remove non-viable cells and activate reparative mechanisms to prevent myocardial rupture. Myeloid cells are especially important in regulating inflammation after MI. The balance between inflammatory and reparative subsets appears crucial and there is compelling evidence implicating excessive inflammation in the mechanism of remodeling. $(6,7)$.

In the traditional paradigm, ischaemic tissue-resident macrophages contribute to neutrophil and inflammatory monocyte recruitment (8). Next, inflammatory Ly $6 \mathrm{C}^{\text {hi }}$ monocyte-derived M1 macrophages secrete pro-inflammatory cytokines, including IL-6, that mediate apoptosis and matrix degradation $(6,9-13)$. These macrophages later shift to a reparative $M 2$ phenotype, secrete anti-inflammatory cytokines, and instigate myocardial repair (14-16). Single cell (sc) RNA-seq experiments have implicated cardiac resident macrophages (RMs) in mediating the inflammatory response to cardiac injury (17-19). C-C chemokine receptor 2expressing $\left(\mathrm{CCR}^{+}\right) \mathrm{RMs}$ (derived from recruited monocytes) and CCR2- RMs (maintained independent of monocyte recruitment) promote and inhibit, respectively, monocyte recruitment after MI (20). However, leukocyte signalling after MI is not fully understood.

One promising candidate pathway involves the transcription factor nuclear factor erythroidderived 2-like 2 (Nrf2). Nrf2 regulates a network of cytoprotective genes (Nrf2-regulated genes, NRGs) and appears to reduce infarct size in models of ischaemia-reperfusion injury (21-24). Additionally, global Nrf2 induction and deletion protect against and exacerbate, respectively, remodeling after $\mathrm{MI}$ with permanent left coronary artery (LCA) ligation $(25,26)$. Previous studies have linked the protective effects of Nrf2 to its antioxidant actions (27); however, Nrf2 has been shown to have anti-inflammatory effects in other settings. Nrf2 also impedes transcriptional upregulation of pro-inflammatory cytokines in M1 macrophages (28), and both global and leukocyte-specific Nrf2 deletion exacerbate inflammation in various murine models, including sepsis and emphysema (29-33). The gene encoding Nrf2 ( $N f e 2 / 2$ ) has been shown by scRNA-seq to be enriched in inflammatory macrophages of murine atherosclerotic aortas, but there was also clear expression in other subsets, indicating a complex contribution to leukocyte biology (34). The role of Nrf2 in regulating the 
inflammatory response after $\mathrm{Ml}$ is unknown. The aim of this study was to investigate the contribution of $\mathrm{Nrf2}$ in leukocytes to the inflammatory response after MI.

\section{Methods}

Detailed methods are provided in Supplemental methods. All use of animals was in accordance with the United Kingdom (Scientific Procedures) Act 1986 (PPL70/8889) and institutional guidelines. Permanent LCA or sham ligation was performed on adult female $\mathrm{Nrf2}^{\%}$ mice and $\mathrm{Nrf2}^{\mathrm{fl} / \mathrm{fl}}$ (WT) littermates both on a C57BL/6J background (35). Nrf2 expression was assessed by Western blot, immunofluorescence, and QRT-PCR of canonical Nrf2-regulated genes. Infarct size was quantified as a proportion of area at risk after 24? recovery. In separate experiments, qRT-PCR was used to quantify mRNA expression in the infarct region after $72 \mathrm{~h}$ of recovery, and fluorescence-activated cell sorting (FACS) of blood and heart digests was performed to examine the cellular inflammatory phenotype.

For RNA sequencing analysis, NRGs were defined according to published chromatin immunoprecipitation sequencing (ChIP-seq) experiments using models of constitutive nuclear accumulation (Keap1 ${ }^{-/-}$) or depletion $\left(\mathrm{Nrf2}^{-/-}\right.$) of Nrf2 (36). To look for an NRG signature in leukocytes, these were cross-referenced with publicly available bulk RNA-seq data from various adult cardiac cell populations from mice subjected to permanent LCA ligation or sham surgery (GEO accession GSE95755 (37)). Gene ontology (GO) analysis was performed with MetaCore ${ }^{\mathrm{TM}}$. To investigate whether the Nrf2-regulated response is a function of specific leukocyte subsets, we used publicly available scRNA-seq data of CD45 cells taken from infarcted tissue $4 \mathrm{~d}$ after $\mathrm{MI}$, chosen to align with FACS experiments (GEO accession GSE106473 (38)). Unbiased clusters were generated and identified using the Immunological Genome (ImmGen) compendium and canonical subset markers. We calculated an NRG score from the summed expression of the 10 most highly expressed NRGs.

Results were compared using a two-tailed t-test or Mann-Whitney $U$ test (non-parametric) for 2 groups of continuous variables, and analysis of variance (ANOVA) and Tukey's Multiple Comparison Test for 3 or more groups. Data is presented as mean[t]SEM, and $p<0.05$ was considered significant. 


\section{Results}

Global $\mathrm{Nrf2}^{-/}$mice display excessive le ukocyte recruitment after $\mathrm{MI}$

Global $\mathrm{Nrf}^{-\%}$ mice have been phenotypically well characterized under baseline conditions $(39,40)$. To assess the effects of Nrf2 independent of confounding changes in infarct size, we employed a permanent ligation model. After $24 \mathrm{~h}$ of experimental LCA ligation, Nrf2 protein levels were significantly increased compared to sham ligation in WT mice (Supplemental Fig. 1A). Heart macrophages ( $C D 68^{+}$cells) in the infarcted area showed higher nuclear translocation of $\mathrm{Nrf2}$ after $\mathrm{Ml}$, which was lost in $\mathrm{Nrf2}^{-\%}$ mice (Supplemental Fig. 1B). Accordingly, hearts of $\mathrm{Nrf}^{-\%}$ mice displayed diminished expression of $\mathrm{Nfe} 2 / 2$ and canonical Nrf2-regulated genes in the heart (Supplemental Fig. 1C-D). Infarct size as a proportion of area at risk was no different between WT and $\mathrm{Nrf2}^{-/-}$mice at $24 \mathrm{~h}$ (WT 93.0 $\pm 1.3 \%$ vs. Nrf2 ${ }^{-1}$ $88.3 \pm 7.9 \%$, Supplemental Fig. $1 E-G)$.

Next, we investigated the inflammatory response to MI. We chose a time point of $72 \mathrm{~h}$, which has been shown to coincide with peak monocyte infiltration (41). The infarct zone from WT and $\mathrm{Nrf2}^{-/}$mice (Fig 1A), or a respective area of left ventricle (LV) from sham controls, and peripheral blood samples were analysed by FACS (gating strategy, Fig. 1B-C). While no differences were apparent in blood at this timepoint (Supplemental Fig. 2), there was a profoundly enhanced inflammatory response in infarcted tissue of $\mathrm{Nrf}^{-\%}$ compared to WT mice (Fig. 1). This comprised total leukocytes (defined as $\mathrm{CD}^{\circ} 5^{+}$) and key myeloid subsets, including neutrophils (CD45 ${ }^{+} \mathrm{CD} 19^{-} \mathrm{Ly}^{-} \mathrm{G}^{+}$) and monocytes (CD45 ${ }^{+} \mathrm{CD} 19^{-}$Ly6G $^{-}$Ly6C $\mathrm{C}^{\text {hi }}$ ) (Fig. 1D-E). Furthermore, there was a more pro-inflammatory (CD206', N1) than proresolution $\left(\mathrm{CD} 26^{+}, \mathrm{N} 2\right)$ neutrophil phenotype. However, there was no significant expansion of monocyte-derived or -independent RMs (defined as CD45 $5^{+} \mathrm{Ly} 6 \mathrm{C}^{\mathrm{lo} C C R 2^{+}}$and $\mathrm{CCR2}$, respectively). Intracellular staining demonstrated increased protein expression of inflammatory cytokines and chemokines, IL-6, iNOS, CD11b and CCR2, in cardiac macrophages (Fig. 1F). This is consistent with the finding that infarcted $\mathrm{Nrf2}^{-/}$hearts also had increased mRNA expression of the chemokine ( $C-C$ motif) ligand 2 (Cc/2/Mcp1) and the chemokine receptor, Cxcr4 (Fig. 1F), which contributes to leukocyte recruitment to the infarcted region. Taken together, these results indicate an inhibitory function of Nrf2 on the inflammatory response after experimental MI. 


\section{Differential NRG expression in leukocytes after experimental MI}

We next wished to determine whether NRGs are relevant in leukocytes themselves. For this, publicly available bulk RNA-seq data from adult mice on day 4 after permanent LCA ligation or sham surgery was analysed (38). Selected results from this dataset were first validated by performing qRT-PCR on our own experimental infarct tissue obtained at day 3 after permanent LCA occlusion or sham in WT mice, finding increased expression of Hmox1, II10, 116, Sod2 and Prdx3 compared to sham (Fig. 2A). We next cross-referenced gene expression data with published ChIP-seq data for Nrf2 targets (36) and found 209 differentially expressed NRGs in leukocytes, compared to 45,60 , and 24 in cardiac myocytes, fibroblasts, and endothelial cells, respectively (Fig. 2B). Clustering of pathway enrichment analysis using gene ontology $(\mathrm{GO})$ annotations highlighted canonical pathways in leukocytes relating to regulation of fibrosis via the TGF- $\beta$ superfamily (Fig. 2 C). This is consistent with the concept that leukocyte NRG expression contributes to the regulation of ventricular remodeling after MI.

\section{$N R G$ expression is a feature of $C C R 2^{+}$cardiac monocytes and macrophages}

We used publicly available scRNA-seq data from $n=1,858 \mathrm{CD}^{+} 5^{+}$sorted leukocytes taken from the infarcts of 4 WT mice at day 4 after Ml, and $n=703$ unsorted cells from the corresponding area of a single, non-infarcted WT heart (38). Unbiased clustering of MI leukocytes generated 11 clusters, which were identified using ImmGen and canonical markers (Fig. 3A; Supplemental Figs. 3-14) and annotated for Nfe2/2 expression (Fig. 3B). Based on the observation that cardiac RMs are central role to leukocyte recruitment after $\mathrm{MI}$, an NRG score was calculated from the summed expression of the most highly expressed NRGs in bioinformatically isolated cardiac monocytes and macrophages (Fth1, Ctsb, Ftl1, Dusp1, Mpeg1, Lcp1, Ubc, Hmox1, Fn1, Cebpb). The global NRG score, applied to MI and WT datasets, was significantly increased in $\mathrm{Ml}$ compared to untreated mice $(\mathrm{p}<0.0001$, Fig. 3C). Further analysis of NRG expression revealed significantly higher expression in $\mathrm{CCR}^{+}$ monocytes and macrophages ( $p<0.0001$, Fig. 3D). Expression of individual NRGs according to cell cluster are shown in Supplemental Fig. 15.

\section{Discussion}

In summary, we demonstrate a robust pro-inflammatory phenotype in global $\mathrm{Nrf2}^{-/}$mice after $\mathrm{Ml}$, encompassing significantly increased pro-inflammatory leukocytes, cytokines, 
leukocyte-recruitment chemokines, and their receptors. Analysis of bulk RNA-seq data confirmed differential NRG expression in leukocytes after MI in WT mice, suggesting that Nrf2 contributes to the inflammatory response, and indicated functions relating to fibrosis and myocardial remodelling. More detailed analyses of scRNA-seq data showed upregulation of NRGs in MI compared to WT samples, which was especially pronounced in $\mathrm{CCR}^{+}$monocytes and macrophages.

The prominent inflammatory phenotype in $\mathrm{Nrf2}^{-\%}$ mice after $\mathrm{Ml}$ was not confined to any leukocyte subset and comprised both neutrophils and macrophages. Previous studies have associated $\mathrm{MI}$ in global $\mathrm{Nrf}^{-/}$mice with worse ventricular remodeling and LV function (26); however, this has previously been related to antioxidant and cytoprotective effects (27), and the extent and cellular composition of the inflammatory phenotype has not been described before, despite the known roles of Nrf2 in mediating inflammation $(28,42)$. Furthermore, hearts of $\mathrm{Nrf2}^{-}$mice had higher expression of chemokines and chemokine receptors. This included higher $\mathrm{Cxcr} 4$ but not $\mathrm{Cxcr} 2$ mRNA expression, which may suggest aged neutrophils with a highly reactive phenotype (43), or recruitment of other CXCR4-expressing leukocyte subsets via stromal derived factor- $1 \alpha$ (SDF- $1 \alpha)$ expression $(44,45)$.

Using RNA-seq data, we confirmed Nfe2/2 and NRG expression in leukocytes, and identified more differentially expressed NRGs compared to cardiac myocytes, fibroblasts, and endothelial cells. Our finding of upregulated RGs after $\mathrm{MI}$ is consistent with previous studies (25). We also observed significantly higher NRG expression in $\mathrm{CCR}^{+}$monocytes and macrophages, which may indicate an important role in monocyte-derived RMs. This contrasts with previous studies that suggest NRG expression is limited to tissue-resident CCR2 cardiac RMs after MI (42). This may relate to how NRGs are selected and indicate distinct functions in diverse subsets. This is relevant because CCR2 ${ }^{+}$and CCR2 cells have respectively negative and positive effects in several models of myocardial injury, including $\mathrm{MI}$, and understanding the contribution of Nrf2 signalling may reveal novel therapeutic targets.

These data support the concept that Nrf2 suppresses inflammation, possibly by impeding over-recruitment of inflammatory cells. Targeting Nrf2 is challenging because it is expressed in several cell types and studies suggest the timing of Nrf2 upregulation is crucial, with most indicating a protective effect of acute expression but harmful effects at later timepoints (46, 47). Therefore, deciphering signaling pathways in the heart, together with identifying novel NRG-expressing leukocyte subsets, may identify novel therapeutic targets. 
Persistently high mortality among patients with post-MI HF necessitates novel approaches. Several clinical trials evaluating non-specific immunosuppression have failed to ameliorate $\mathrm{HF}$ after MI (48), which is unsurprising given the complex relationship between inflammatory and reparative processes. Approaches specifically targeting detrimental inflammation are required. The CANTOS trial showed that targeting atherosclerosis with canakinumab, an anti-IL-1 $\beta$ monoclonal antibody, reduced major adverse cardiac events in high-risk patients (49). After $\mathrm{Ml}$, the anti-inflammatory agent colchicine reduced ischaemic cardiovascular events compared to placebo (50). These provide the first evidence of targeted approaches improving outcomes in cardiovascular disease. Our findings are clinically relevant as we demonstrate that Nrf2 in leukocytes has a fundamental role in cardiac inflammation after Ml. Therefore, targeting acute inflammation via Nrf2-regulated pathways may be one such approach to preventing HF after MI. 
bioRxiv preprint doi: https://doi.org/10.1101/2022.01.10.475615; this version posted January 10, 2022. The copyright holder for this preprint (which was not certified by peer review) is the author/funder. All rights reserved. No reuse allowed without permission.

\section{Acknowledgements}

The authors wish to thank Matteo Beretta for his assistance with confocal imaging. 


\section{References}

1. Velagaleti RS, Pencina MJ, Murabito JM, Wang TJ, Parikh NI, D'Agostino $\mathrm{RB}$, et al. Long-term trends in the incidence of heart failure after myocardial infarction. Circulation. 2008;118(20):2057-62.

2. Gerber Y, Weston SA, Berardi C, McNallan SM, Jiang R, Redfield MM, et al. Contemporary trends in heart failure with reduced and preserved ejection fraction after myocardial infarction: a community study. Am J Epidemiol. 2013;178(8):1272-80.

3. Kaul P, Ezekowitz JA, Armstrong PW, Leung BK, Savu A, Welsh RC, et al. Incidence of heart failure and mortality after acute coronary syndromes. Am Heart J. 2013;165(3):379-85 e2.

4. McManus DD, Chinali M, Saczynski JS, Gore JM, Yarzebski J, Spencer FA, et al. 30-year trends in heart failure in patients hospitalized with acute myocardial infarction. Am J Cardiol. 2011;107(3):353-9.

5. Gerber Y, Weston SA, Enriquez-Sarano M, Berardi C, Chamberlain AM, Manemann SM, et al. Mortality Associated With Heart Failure After Myocardial Infarction: A Contemporary Community Perspective. Circ Heart Fail. 2016;9(1):e002460.

6. Frangogiannis NG. Inflammation in cardiac injury, repair and regeneration. Curr Opin Cardiol. 2015;30(3):240-5.

7. van der Laan AM, Hirsch A, Robbers LF, Nijveldt R, Lommerse I, Delewi R, et al. A proinflammatory monocyte response is associated with myocardial injury and impaired functional outcome in patients with ST-segment elevation myocardial infarction: monocytes and myocardial infarction. Am Heart J. 2012;163(1):57-65 e2.

8. Leuschner F, Rauch PJ, Ueno T, Gorbatov R, Marinelli B, Lee WW, et al. Rapid monocyte kinetics in acute myocardial infarction are sustained by extramedullary monocytopoiesis. J Exp Med. 2012;209(1):123-37.

9. Kobara M, Noda K, Kitamura M, Okamoto A, Shiraishi T, Toba H, et al. Antibody against interleukin-6 receptor attenuates left ventricular remodelling after myocardial infarction in mice. Cardiovasc Res. 2010;87(3):424-30.

10. Bujak M, Dobaczewski M, Chatila K, Mendoza LH, Li N, Reddy A, et al. Interleukin-1 receptor type I signaling critically regulates infarct healing and cardiac remodeling. Am J Pathol. 2008;173(1):57-67.

11. Ohtsuka T, Hamada M, Inoue K, Ohshima K, Sujzuki J, Matsunaka T, et al. Relation of circulating interleukin- 6 to left ventricular remodeling in patients with reperfused anterior myocardial infarction. Clin Cardiol. 2004;27(7):417-20.

12. Deten A, Volz HC, Briest W, Zimmer HG. Cardiac cytokine expression is upregulated in the acute phase after myocardial infarction. Experimental studies in rats. Cardiovasc Res. 2002;55(2):329-40.

13. Kaminski KA, Kozuch M, Bonda T, Wojtkowska I, Kozieradzka A, Dobrzycki S, et al. Coronary sinus concentrations of interleukin 6 and its soluble receptors are affected by reperfusion and may portend complications in patients with myocardial infarction. Atherosclerosis. 2009;206(2):581-7.

14. Walter W, Alonso-Herranz L, Trappetti V, Crespo I, Ibberson M, Cedenilla $M$, et al. Deciphering the Dynamic Transcriptional and Post-transcriptional Networks of Macrophages in the Healthy Heart and after Myocardial Injury. Cell Rep. 2018;23(2):622-36. 
15. Dobaczewski M, Xia Y, Bujak M, Gonzalez-Quesada C, Frangogiannis NG. CCR5 signaling suppresses inflammation and reduces adverse remodeling of the infarcted heart, mediating recruitment of regulatory $\mathrm{T}$ cells. Am J Pathol. 2010;176(5):2177-87.

16. Nahrendorf M, Swirski FK, Aikawa E, Stangenberg L, Wurdinger T, Figueiredo JL, et al. The healing myocardium sequentially mobilizes two monocyte subsets with divergent and complementary functions. J Exp Med. 2007;204(12):3037-47.

17. Wong NR, Mohan J, Kopecky BJ, Guo S, Du L, Leid J, et al. Resident cardiac macrophages mediate adaptive myocardial remodeling. Immunity. 2021;54(9):2072-88 e7.

18. Zaman R, Hamidzada H, Kantores C, Wong A, Dick SA, Wang Y, et al. Selective loss of resident macrophage-derived insulin-like growth factor-1 abolishes adaptive cardiac growth to stress. Immunity. 2021;54(9):2057-71 e6.

19. Dick SA, Macklin JA, Nejat S, Momen A, Clemente-Casares X, Althagafi MG, et al. Self-renewing resident cardiac macrophages limit adverse remodeling following myocardial infarction. Nat Immunol. 2019;20(1):29-39.

20. Bajpai G, Bredemeyer A, Li W, Zaitsev K, Koenig AL, Lokshina I, et al. Tissue Resident CCR2- and CCR2+ Cardiac Macrophages Differentially Orchestrate Monocyte Recruitment and Fate Specification Following Myocardial Injury. Circ Res. 2019;124(2):263-78.

21. Nguyen T, Sherratt PJ, Nioi P, Yang CS, Pickett CB. Nrf2 controls constitutive and inducible expression of ARE-driven genes through a dynamic pathway involving nucleocytoplasmic shuttling by Keap1. J Biol Chem. 2005;280(37):32485-92.

22. Yu H, Shi L, Zhao S, Sun Y, Gao Y, Sun Y, et al. Triptolide Attenuates Myocardial Ischemia/Reperfusion Injuries in Rats by Inducing the Activation of Nrf2/HO-1 Defense Pathway. Cardiovasc Toxicol. 2016;16(4):325-35.

23. Cheng L, Jin Z, Zhao R, Ren K, Deng C, Yu S. Resveratrol attenuates inflammation and oxidative stress induced by myocardial ischemia-reperfusion injury: role of Nrf2/ARE pathway. Int J Clin Exp Med. 2015;8(7):10420-8.

24. Sun G, Li Y, Ji Z. Atorvastatin attenuates inflammation and oxidative stress induced by ischemia/reperfusion in rat heart via the Nrf2 transcription factor. Int J Clin Exp Med. 2015;8(9):14837-45.

25. Shimizu Y, Nicholson CK, Lambert JP, Barr LA, Kuek N, Herszenhaut D, et al. Sodium Sulfide Attenuates Ischemic-Induced Heart Failure by Enhancing Proteasomal Function in an Nrf2-Dependent Manner. Circ Heart Fail. 2016;9(4):e002368.

26. Strom J, Chen QM. Loss of Nrf2 promotes rapid progression to heart failure following myocardial infarction. Toxicol Appl Pharmacol. 2017;327:52-8.

27. Ashrafian H, Czibik G, Bellahcene M, Aksentijevic D, Smith AC, Mitchell SJ, et al. Fumarate is cardioprotective via activation of the Nrf2 antioxidant pathway. Cell Metab. 2012;15(3):361-71.

28. Kobayashi EH, Suzuki T, Funayama R, Nagashima T, Hayashi M, Sekine H, et al. Nrf2 suppresses macrophage inflammatory response by blocking proinflammatory cytokine transcription. Nat Commun. 2016;7:11624.

29. Itoh K, Mochizuki M, Ishii Y, Ishii T, Shibata T, Kawamoto Y, et al. Transcription factor Nrf2 regulates inflammation by mediating the effect of 15deoxy-Delta(12,14)-prostaglandin j(2). Mol Cell Biol. 2004;24(1):36-45. 
30. Ishii $\mathrm{Y}$, Itoh $\mathrm{K}$, Morishima $\mathrm{Y}$, Kimura $\mathrm{T}$, Kiwamoto $\mathrm{T}$, Iizuka $\mathrm{T}$, et al. Transcription factor Nrf2 plays a pivotal role in protection against elastaseinduced pulmonary inflammation and emphysema. J Immunol. 2005;175(10):6968-75.

31. Iizuka T, Ishii Y, Itoh K, Kiwamoto T, Kimura T, Matsuno Y, et al. Nrf2deficient mice are highly susceptible to cigarette smoke-induced emphysema. Genes Cells. 2005;10(12):1113-25.

32. Thimmulappa RK, Lee H, Rangasamy T, Reddy SP, Yamamoto M, Kensler TW, et al. Nrf2 is a critical regulator of the innate immune response and survival during experimental sepsis. J Clin Invest. 2006;116(4):984-95.

33. Kong X, Thimmulappa R, Craciun F, Harvey C, Singh A, Kombairaju P, et al. Enhancing Nrf2 pathway by disruption of Keap1 in myeloid leukocytes protects against sepsis. Am J Respir Crit Care Med. 2011;184(8):928-38.

34. Cochain C, Vafadarnejad E, Arampatzi P, Pelisek J, Winkels H, Ley K, et al. Single-Cell RNA-Seq Reveals the Transcriptional Landscape and Heterogeneity of Aortic Macrophages in Murine Atherosclerosis. Circ Res. 2018;122(12):1661-74.

35. Protti A, Dong X, Sirker A, Botnar R, Shah AM. MRI-based prediction of adverse cardiac remodeling after murine myocardial infarction. Am J Physiol Heart Circ Physiol. 2012;303(3):H309-14.

36. Malhotra D, Portales-Casamar E, Singh A, Srivastava S, Arenillas D, Happel $\mathrm{C}$, et al. Global mapping of binding sites for Nrf2 identifies novel targets in cell survival response through ChIP-Seq profiling and network analysis. Nucleic Acids Res. 2010;38(17):5718-34.

37. Quaife-Ryan GA, Sim CB, Ziemann M, Kaspi A, Rafehi H, Ramialison M, et al. Multicellular Transcriptional Analysis of Mammalian Heart Regeneration. Circulation. 2017;136(12):1123-39.

38. King KR, Aguirre AD, Ye YX, Sun Y, Roh JD, Ng RP, Jr., et al. IRF3 and type I interferons fuel a fatal response to myocardial infarction. Nat Med. 2017;23(12):1481-7.

39. Brewer AC, Murray TV, Arno M, Zhang M, Anilkumar NP, Mann GE, et al. Nox4 regulates Nrf2 and glutathione redox in cardiomyocytes in vivo. Free Radic Biol Med. 2011;51(1):205-15.

40. Smyrnias I, Zhang X, Zhang M, Murray TV, Brandes RP, Schroder K, et al. Nicotinamide adenine dinucleotide phosphate oxidase-4-dependent upregulation of nuclear factor erythroid-derived 2-like 2 protects the heart during chronic pressure overload. Hypertension. 2015;65(3):547-53.

41. Peet C, Ivetic A, Bromage DI, Shah AM. Cardiac monocytes and macrophages after myocardial infarction. Cardiovasc Res. 2020;116(6):1101-12.

42. Calcagno DM, Ng RP, Jr., Toomu A, Zhang C, Huang K, Aguirre AD, et al. The myeloid type I interferon response to myocardial infarction begins in bone marrow and is regulated by Nrf2-activated macrophages. Sci Immunol. 2020;5(51).

43. Uhl B, Vadlau Y, Zuchtriegel G, Nekolla K, Sharaf K, Gaertner F, et al. Aged neutrophils contribute to the first line of defense in the acute inflammatory response. Blood. 2016;128(19):2327-37.

44. Bromage DI, Taferner S, He Z, Ziff OJ, Yellon DM, Davidson SM. Stromal cell-derived factor-1alpha signals via the endothelium to protect the heart against ischaemia-reperfusion injury. J Mol Cell Cardiol. 2019;128:187-97. 
45. Bromage DI, Davidson SM, Yellon DM. Stromal derived factor 1alpha: a chemokine that delivers a two-pronged defence of the myocardium. Pharmacol Ther. 2014;143(3):305-15.

46. Rajasekaran NS, Varadharaj S, Khanderao GD, Davidson CJ, Kannan S, Firpo MA, et al. Sustained activation of nuclear erythroid 2-related factor 2/antioxidant response element signaling promotes reductive stress in the human mutant protein aggregation cardiomyopathy in mice. Antioxid Redox Signal. 2011;14(6):957-71.

47. Kensler TW, Wakabayashi N. Nrf2: friend or foe for chemoprevention? Carcinogenesis. 2010;31(1):90-9.

48. Rymer JA, Newby LK. Failure to Launch: Targeting Inflammation in Acute Coronary Syndromes. JACC Basic Transl Sci. 2017;2(4):484-97.

49. Ridker PM, Everett BM, Thuren T, MacFadyen JG, Chang WH, Ballantyne C, et al. Antiinflammatory Therapy with Canakinumab for Atherosclerotic Disease. N Engl J Med. 2017;377(12):1119-31.

50. Tardif JC, Kouz S, Waters DD, Bertrand OF, Diaz R, Maggioni AP, et al. Efficacy and Safety of Low-Dose Colchicine after Myocardial Infarction. N Engl J Med. 2019;381(26):2497-505. 


\section{Figure legends}

Fig. 1: $\mathrm{Nrf2}^{\%}$ mice have a pro-inflammatory phenotype after MI.

9-13 week old mice were anaesthetised and subjected to permanent LAD ligation prior to recovery for 72 h. A. Schematic of Ml experiment in which the infarct zone was isolated from excised hearts; B. Gating strategy for FACS analysis (1-3, Ly6C $C^{\text {, }}$, Ly6C $C^{\text {lo }}$ and Ly6C', respectively, monocytes and macrophages; 4, neutrophils); C. Representative plots from FACS analysis showing higher leukocyte infiltration in $\mathrm{Nrf2}^{-/}$mice after MI; D. Quantification of leukocytes in heart digests, defined as (left to right): $\mathrm{CD} 45^{+}$(total leukocytes), $\mathrm{CD} 45^{+} \mathrm{CD} 19^{-}$ Ly6G $^{+}$CD206- (N1) and CD45 CD19-Ly6GCD206 (N2) neutrophils (top), and monocytes

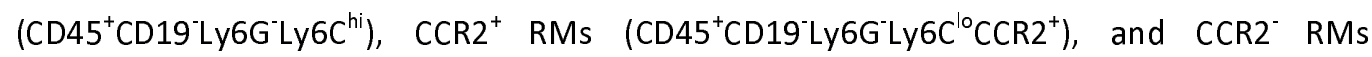
(CD45 ${ }^{+} \mathrm{CD}^{-}$Ly6G $^{-}$Ly6C $^{\mathrm{lo}} \mathrm{CCR2}$ ) (bottom). Data shown as cells/mg, $\mathrm{n}=5-8$ per group compared using 1-way ANOVA with Tukey's post-test; E. Representative histograms from Ly6C ${ }^{\text {hi }}$ cells showing expression of inflammatory modulators, iNOS, CD11b, and IL-6; F. Expression in the infarct region in WT mice $(n=10)$, compared to $\mathrm{Nrf2}^{\%}$ mice $(n=12-13)$. Data presented as mean \pm SEM and compared using a t-test. ${ }^{*} p<0.05, * * p<0.01, * * * p<0.001$.

Fig. 2: Myocardial infarction activates Nrf2-dependent signalling in leukocytes.

A. Validation of publicly available bulk RNAseq data; Hmox1, /110, Ccl2, Cxcr2, Ccr2, Sod2, $\operatorname{Prdx3}$ and $/ 16$ expression in the infarct region on D3 after MI ( $n=9-10)$, compared to sham in WT mice $(n=4-6)$. Data presented as mean \pm SEM and compared using a t-test. ${ }^{*} p<0.05$, $*^{*} p<0.01, * * * p<0.001$; B. Volcano plots showing differentially expressed Nrf2-regulated genes in different cell types day 3 after MI or sham surgery in adult mice (using publicly available data). $\mathbf{N}$ represents the number of differentially expressed Nrf2-regulated genes; $\mathbf{C}$. Gene enrichment analysis of GO pathways relating to Nrf2-regulated genes.

Fig 3: Nrf2-regulated gene (NRG) expression in CCR2+ cardiac monocytes and macrophages.

A. Publicly available single-cell RNA-seq data for 1,858 single cells isolated from a single WT mouse $4 \mathrm{~d}$ after permanent LCA occlusion, clustered and displayed on a uniform manifold approximation and projection (UMAP) plot. Cell lineage inferred by marker gene signatures; B. UMAPs annotated with Nfe2I2 expression; C. Global Nrf2-regulated gene (NRG) score based on summed expression of NRGs in MI and WT datasets, normalized to cell number; $\mathbf{D}$. NRG score in $\mathrm{CCR}^{-}$and $\mathrm{CCR}^{+}$monocytes and macrophages, normalized to cell number. Data presented as mean \pm SEM and compared using the Mann-Whitney test, $* * * * p<0.0001$. 
mice have a pro-inflammatory phenotype after MI

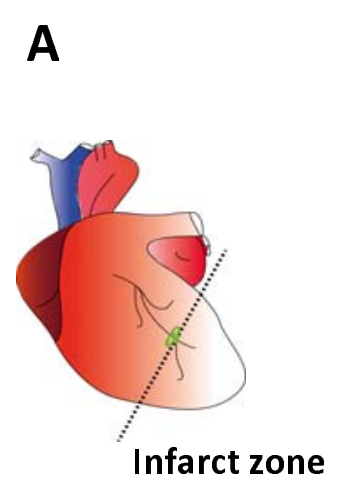

B
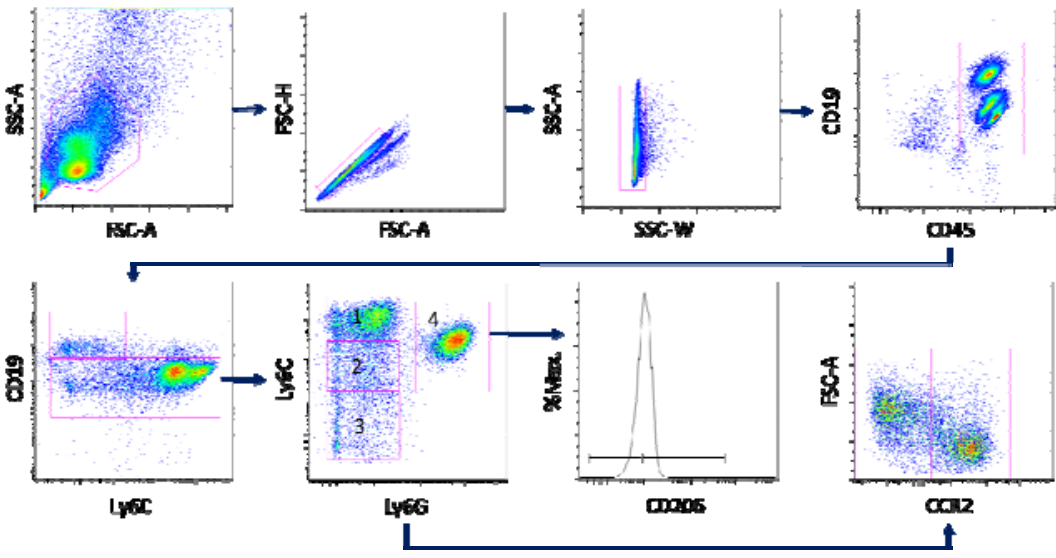

C
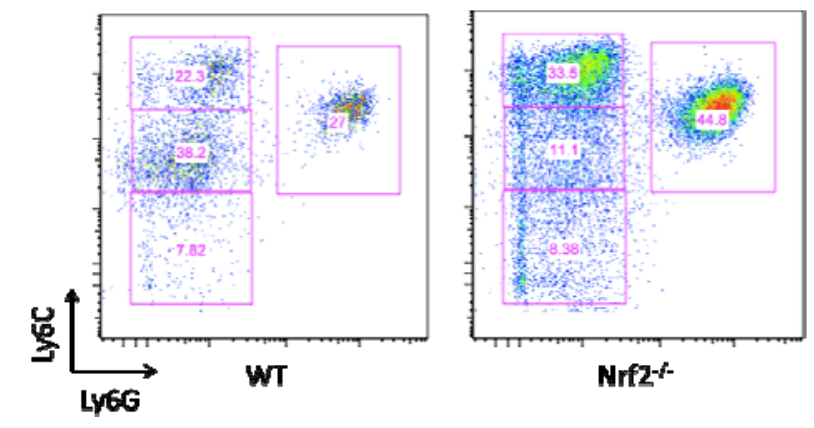

D
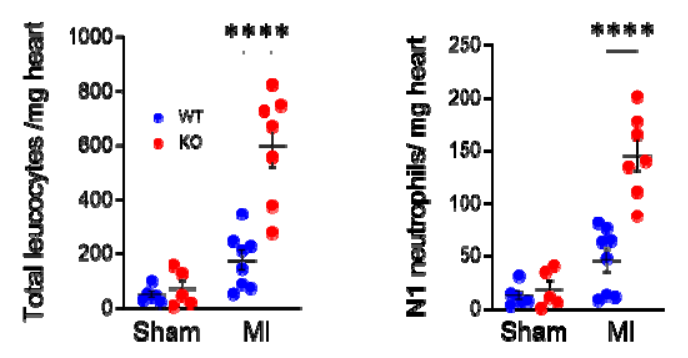

E
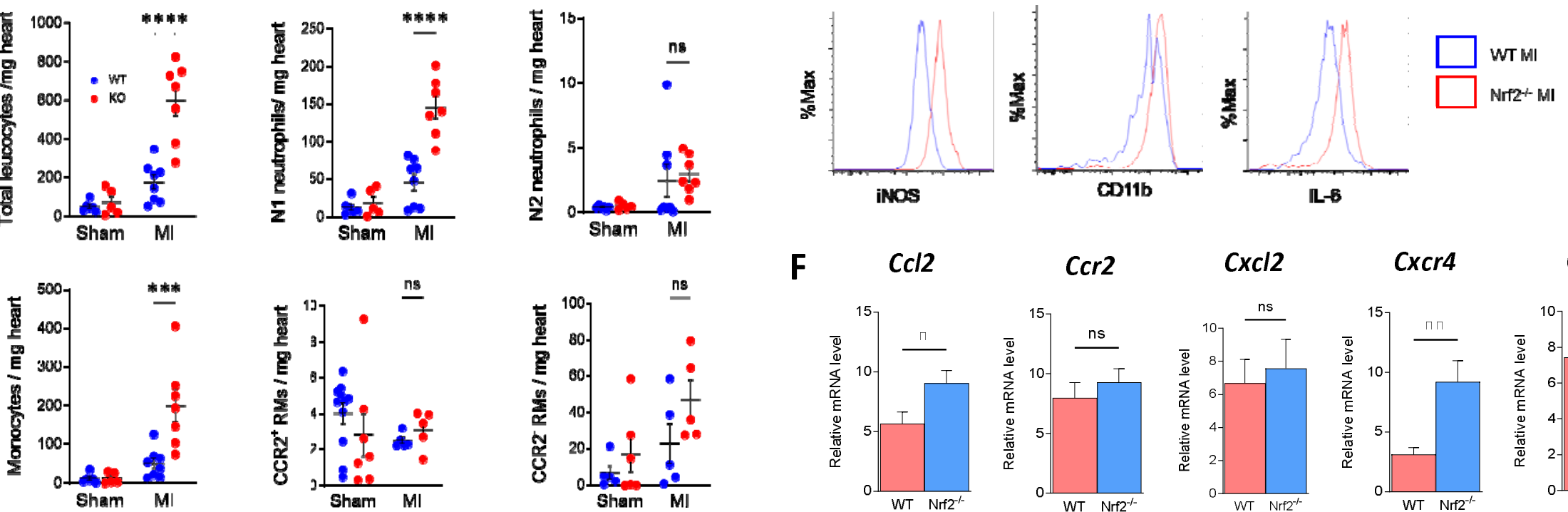

F $\quad \mathrm{Ccl} 2$
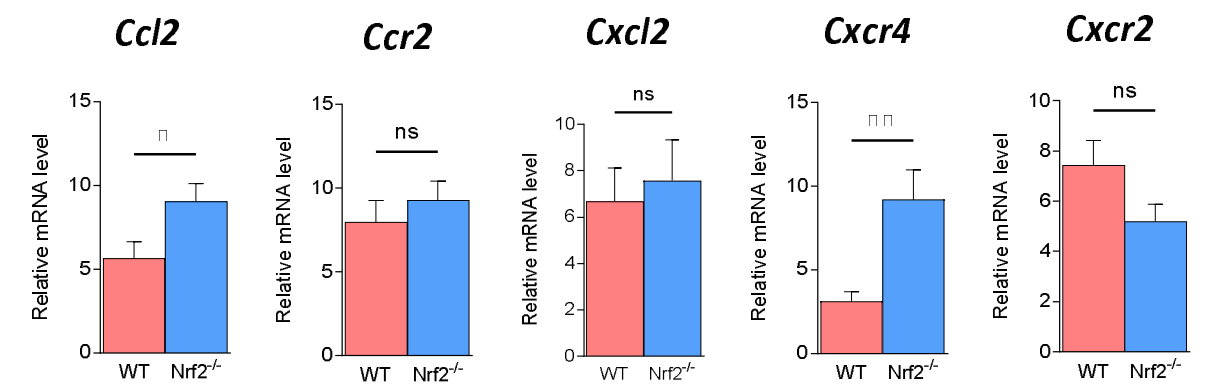
rdial infarction activates Nrf2-dependent signalling in leukocytes

A Hmox1

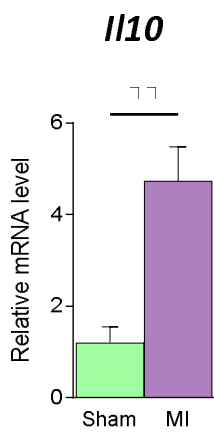

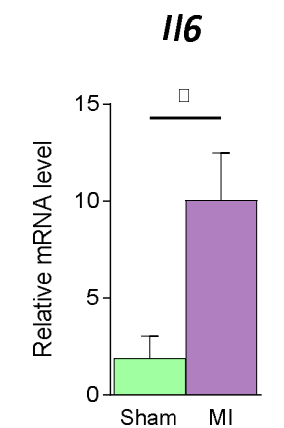

116

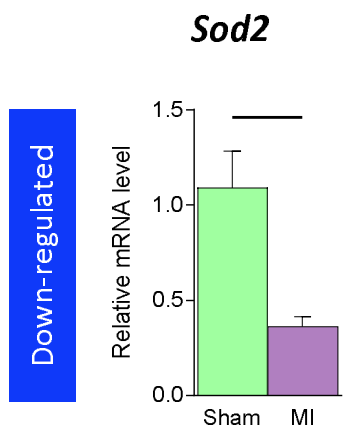

Sod2

$\operatorname{Prdx3}$

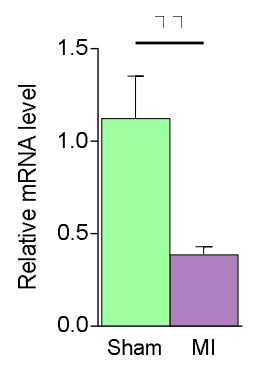

B

Myocytes

Fibroblasts

Leukocytes

Endothelial cells

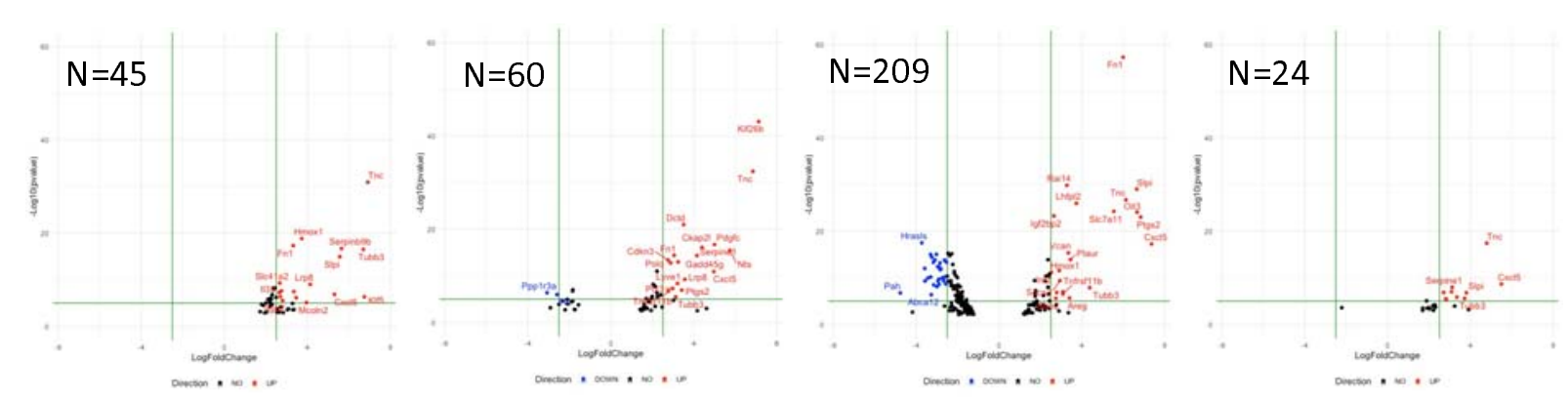

C

Development_Regulation of epithelial-to-mesenchyman transition (EMT)

Signal transduction_WNT/Beta-catenin signalling in tissue homeostasis NRF2 regulation of oxidative stress response

Development TGF-beta-dependent induction of EMT via SMADs

Signal transduction_BMP signaling via ALK-4 and TGF-beta receptor type I
Cell adhesion_Gap junctions

Development_Androgen receptor in reproductive system development

Cell cycle Role of Nek in cell cycle regulation

Role of TGF-beta 1 in fibrosis development after myocardial infarction

Signal transduction_BMP signaling via BMPR1A and BMPR1B receptors

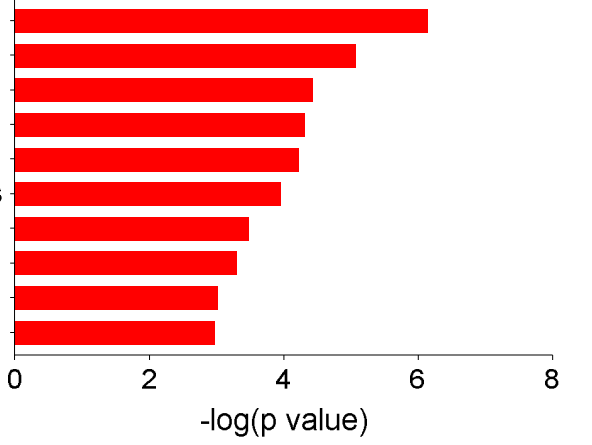


gulated gene (NRG) expression in CCR2+ cardiac monocytes and macrophages

A

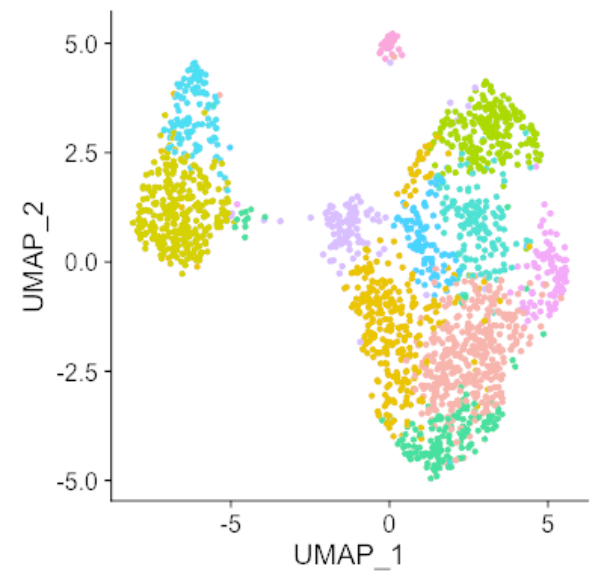

C

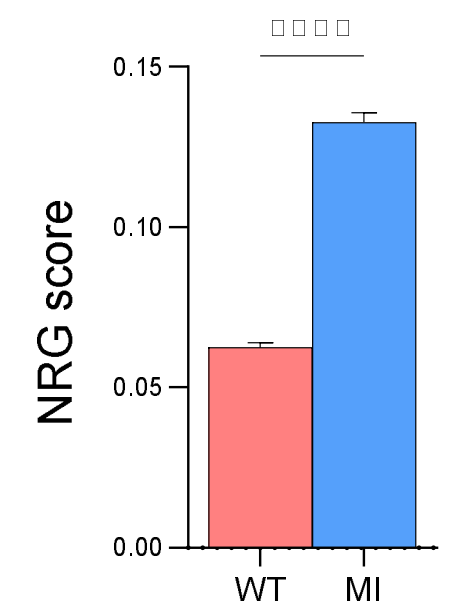

Macrophage 1

- Macrophage 2

- Granulocyte 1

- Macrophage 3

- Macrophage 4

- Dendritlc cell

- Granulocyte 2

Macrophage 5

- Monocyte

Prollferating

- T/NKcell

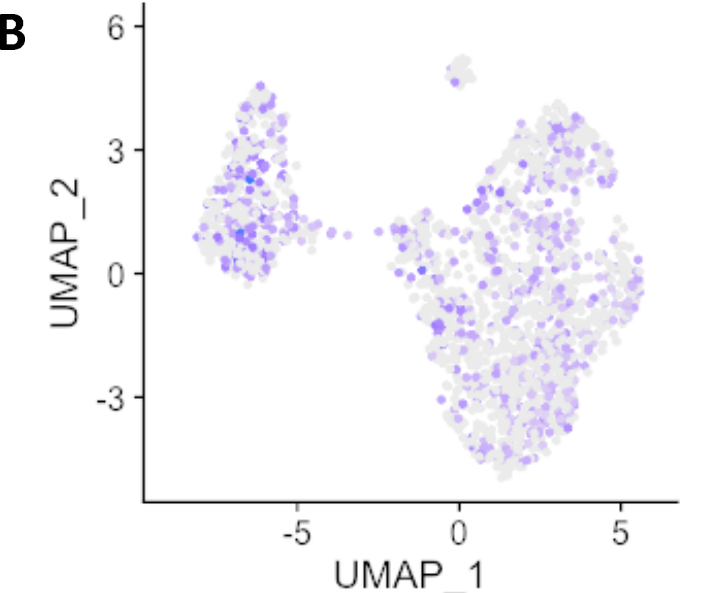

D

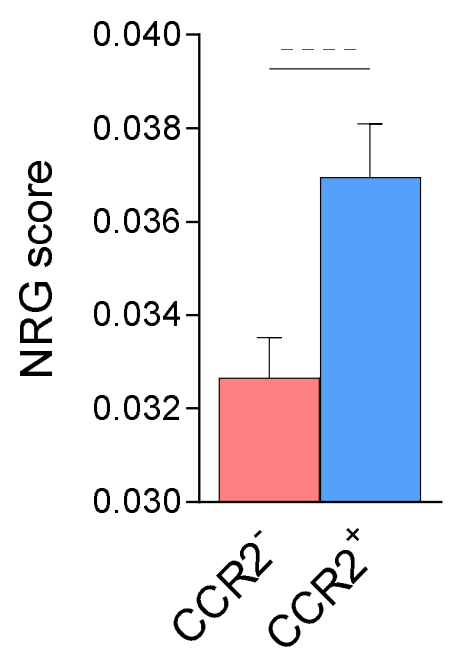

